\title{
Thought Suppression, Impaired Regulation of Urges, and Addiction-Stroop Predict Affect-Modulated Cue-Reactivity among Alcohol Dependent Adults
}

\author{
Eric L. Garland \\ Trinity Institute for the Addictions, College of Social Work, Florida State University \\ Kristin Carter and Katie Ropes \\ College of Social Work, Florida State University \\ Matthew O. Howard \\ University of North Carolina at Chapel Hill
}

\begin{abstract}
Abstinent alcohol dependent individuals commonly employ thought suppression to cope with stress and intrusive cognitions about alcohol. This strategy may inadvertently bias attention toward alcohol-related stimuli while depleting neurocognitive resources needed to regulate urges, manifested as decreased heart rate variability (HRV) responsivity to alcohol cues. The present study tested the hypothesis that trait and state thought suppression, impaired regulation of urges, and alcohol attentional bias as measured by the Addiction-Stroop would have significant effects on the HRV responsivity of 58 adults in residential treatment for alcohol dependence (mean age $=$ $39.6 \pm 9.4,81 \%$ female) who participated in an affect-modulated cue-reactivity protocol.

Regression analyses controlling for age, level of pre-treatment alcohol consumption, and baseline HRV indicated that higher levels of trait thought suppression, impaired regulation of alcohol urges, and attentional fixation on alcohol cues were associated with lower HRV responsivity during stress-primed alcohol cue-exposure. Moreover, there was a significant state $\mathrm{X}$ trait suppression interaction on HRV cue-responsivity, such that alcohol dependent persons reporting high levels of state and trait suppression exhibited less HRV during cue-exposure than persons reporting low levels of state and trait suppression. Results suggest that chronic thought suppression taxes regulatory resources reflected in reduced HRV responsivity, an effect that is particularly evident when high trait suppressors engage in intensive suppression of drinkingrelated thoughts under conditions of stress. Treatment approaches that offer effective alternatives to the maladaptive strategy of suppressing alcohol urges may be crucial for relapse prevention.
\end{abstract}

\section{Keywords}

Alcohol dependence; attentional bias; cue-reactivity; HRV; Stroop; thought suppression

\footnotetext{
(C) 2011 Elsevier B.V. All rights reserved.

Correspondence to: Eric L. Garland, Ph.D. Trinity Institute for the Addictions Florida State University University Center, Building C Tallahassee, Fl 32306-2570 850-645-9571 egarland@ fsu.edu.

Publisher's Disclaimer: This is a PDF file of an unedited manuscript that has been accepted for publication. As a service to our customers we are providing this early version of the manuscript. The manuscript will undergo copyediting, typesetting, and review of the resulting proof before it is published in its final citable form. Please note that during the production process errors may be discovered which could affect the content, and all legal disclaimers that apply to the journal pertain.
} 
Alcohol dependent persons in recovery confront numerous challenges to their resolve to remain abstinent. Exposure to socioenvironmental stressors may render such individuals vulnerable to relapse, as stress promotes habitual behaviors that are resistant to changes in outcome contingencies while inducing deficits in executive function (Dias-Ferreira et al., 2009). Hence, it is plausible that stress enhances relapse risk by evoking automatic appetitive responses and impairing functions involved in the regulation of alcohol urges (Garland, Boettiger, \& Howard, 2011). Although the relationship between stress and alcohol use has been studied for decades (e.g., Conger, 1951; Capel \& Herman, 1972), less is known about how the neurocognitive mediators of the stress response promote relapse among recovering alcohol dependent individuals.

Recently, we proposed a theoretical model that specifies feedback circuits between stress, implicit cognition, self-regulation attempts, and the drive to consume alcohol (Garland et al., 2011). According to this model, repeated alcohol misuse in the context of stress and negative affect may establish automatic alcohol-use action schemas, memory structures that coordinate and compel behaviors involved in alcohol consumption. Hypothetically, these schemas are evoked in a conditioned response to present stressors (Tiffany, 1990), which may bias attention towards alcohol (Field \& Powell, 2007) and increase the urge to drink as a means of palliative coping. However, among alcohol dependent persons in recovery, such urges are often egodystonic, i.e., perceived as intrusive and incongruent with the goal of abstinence (Soutullo, McElroy, \& Goldsmith, 1998). In an attempt to regulate mounting alcohol urges, such individuals may employ "willpower" to suppress the urge to drink. Indeed, the attempt to suppress intrusive thoughts of drinking is common among persons in treatment for alcohol use disorders, and is prospectively associated with greater frequency of alcohol-related thoughts and craving episodes (Kavanaugh, May, \& Andrade, 2009).

Ironically, thought suppression may be a key component in the cognitive-mediation of stress-precipitated relapse insofar as this strategy inadvertently further biases attention toward alcohol-related cognitions and affective reactions. These so-called "rebound effects" are suggested by research demonstrating that attempted suppression often results in an increased rate of the thoughts and emotions it is directed against (Wegner, Schneider, Carter, $\&$ White, 1987; Wenzlaff \& Wegner, 2000). For example, when asked to suppress urges following alcohol cue exposure, alcohol dependent adults exhibited speeded reaction times to alcohol-related statements relative to control phrases (Palfai, Monti, Colby, \& Rohsenow, 1997). Similarly, abstinent alcohol dependent adults experienced greater Stroop interference for the word "alcohol" after they had initially attempted to suppress alcohol-related thoughts compared to individuals who expressed such thoughts (Klein, 2007). Rebound effects have also been identified for appetitive behavior. Experimental induction of suppression of thoughts of smoking (Erskine, Georgiou, \& Kvavilashvili, 2010) and eating (Erskine \& Georgiou, 2010) leads to greater enactment of such behaviors. These findings may be explained by Wegner's ironic process theory (1994), which asserts that suppression involves two processes: a) a conscious search for cognitive contents consistent with the desired mental state, and b) an implicit monitoring process that searches continually for cognitions that are inconsistent with the desired state. When attention is automatically deployed in search of undesirable mental content to be replaced, the ensuing positive feedback loop leads to hyperaccessibility of unwanted cognitions (Wegner \& Erber, 1992), amplifying their frequency and intensity under conditions of stress (Nixon, Cain, Nehmy, \& Seymour, 2009; Williams \& Moulds, 2007). As a result, the intrusive and distressing nature of the target thoughts is magnified by the very process employed to avoid them (Abramowitz, Tolin, \& Street, 2001).

Hypothetically, suppression may exhaust the capacity for self-regulation, which Baumeister and colleagues have characterized as a limited resource that is depleted through repeated 
acts of self-control (Baumeister, 2003; Muraven \& Baumeister, 2000). Integrating notions drawn from Wegner's and Baumeister's theories, we propose that suppression undermines cognitive control ( $\mathrm{Pu}, \mathrm{Schmeichel,} \mathrm{\&} \mathrm{Demaree,} \mathrm{2010),} \mathrm{resulting} \mathrm{in} \mathrm{impaired} \mathrm{regulation} \mathrm{of}$ alcohol urges, increased stress, and relapse. These deleterious effects may be exacerbated when persons whose self-regulatory resources have already been depleted due to chronic thought suppression (Wegner \& Zanakos, 1994) engage in acute or state suppression of alcohol-related thoughts triggered by stressful circumstances.

Although suppression is intended to reduce emotional experience, instead, it often leads to sympathetic arousal, indicated by changes in electrodermal response, finger pulse amplitude, pulse transmission time, and skin temperature (Gross \& Levenson, 1993; Roberts, Levenson, $\&$ Gross, 2008). The physiological effects of suppression may be illuminated by the neurovisceral integration model (Thayer \& Lane, 2000), which proposes that a network of central (e.g., prefrontal and anterior cingulate cortices) and autonomic (e.g., vagal nerve) structures regulate attention and emotion by exerting inhibitory influences over perturbations to visceral homeostasis, such as those experienced by abstinent alcohol dependent persons when confronted with stressors and/or alcohol cues. Regulation of attentional and emotional responses by this central autonomic network may be indicated by increases in high-frequency heart rate variability (HRV), i.e., the beat-to-beat variation in heart rate modulated by parasympathetic outflows of the vagus on the pacemaker of the heart (Thayer \& Lane, 2009). Increased HRV is positively correlated with the ability to flexibly regulate attention and emotion (Friedman, 2007; Thayer, Hansen, Saus-Rose, \& Johnsen, 2009). Conversely, decreased HRV is observed during inflexible cognitiveemotional states associated with heightened and prolonged sympathetic arousal (Brosschot, Gerin, \& Thayer, 2006), such as attentional hypervigilance (Hansen, Johnsen, \& Thayer, 2003) and perseverative cognition (Brosschot, 2010; Key, Campbell, Bacon, \& Gerin 2008). Hence, the neurovisceral integration model predicts that alcohol dependent individuals would exhibit attenuated HRV responsivity coupled with a rigid focus of attention on alcohol cues (indexed by greater alcohol attentional bias) when they employ thought suppression to regulate stress and urges.

Such predictions accord with results from Injaldsson, Laberg, and Thayer's (2003) study of 49 alcohol dependent subjects, which found that chronic thought suppression was inversely associated with heart rate variability (HRV) responsivity to an imaginal alcohol cueexposure script. In addition, individuals who were less able to regulate alcohol urges exhibited reduced HRV responses to alcohol cues compared to persons reporting greater ability to regulate urges, who evidenced elevated HRV in response to alcohol cues. Hence, thought suppression among alcohol dependent individuals is linked with impaired regulation of alcohol urges by the central autonomic network.

Although thought suppression and impaired ability to regulate drinking urges were associated with an attenuated HRV response, Ingjaldsson et al. (2003) did not examine these relationships simultaneously to determine the extent to which these factors independently contributed to HRV responsivity to alcohol cues among alcohol dependent persons. Moreover, no behavioral measures of attention to alcohol cues were evaluated, leaving relationships between alcohol attentional bias (AB), thought suppression, impaired regulation of urges, and HRV responsivity unspecified. Lastly, Ingjaldsson et al. (2003) did not discriminate chronic thought suppression, that is, the trait-like tendency to suppress thoughts in general in everyday life, from state suppression of alcohol-related thoughts during the cue-reactivity paradigm. The present investigation sought to provide a partial test of our theoretical model (Figure 1) and extend the work of Ingjaldsson e al. (2003) by examining these relationships in the context of an affect-modulated cue-reactivity paradigm intended to induce stress prior to alcohol cue-exposure (Cheetham, Allen, Yucel, \& 
Lubman, 2010). The use of this paradigm was crucial, given the centrality of stress to our conceptual framework, and the body of work demonstrating that stress often precipitates relapse (Sinha, 2007) through its effects on cognitive and affective processes in addiction (Garland et al., 2011). We hypothesized that trait suppression, impaired regulation of urges, and alcohol AB would be significantly inversely associated with HRV responsivity to stressprimed alcohol cues, and that higher levels of trait suppression and impaired regulation of alcohol urges would predict elevated subjective alcohol cue-reactivity. We also hypothesized that greater state suppression of thoughts of drinking during stress cueexposure would be associated with increases in subjective reactivity to subsequent alcohol cue-exposure. Finally, we anticipated that state and trait thought suppression would interact significantly to produce attenuation of HRV responsivity to alcohol cues.

\section{METHODS}

\section{Participants}

Fifty-eight alcohol dependent adults (47 men; 11 women) in residential treatment for substance dependence participated in the study. Participants met lifetime Diagnostic and Statistical Manual of Mental Disorders-Fourth Edition (DSM-IV) alcohol dependence criteria (determined via semi-structured psychiatric interview) and had been in residential treatment for $\geq 18$ months. In this treatment program, 18 months marked the time of transition to employment and residence outside of the treatment facility, and thus represents a point of increased relapse risk. The mean age of participants was $39.6(S D=9.4)$. On average, participants had been in residential care for 22.2 months $(S D=3.6)$. Overall, participants met an average of $6.5(S D=1.0)$ DSM-IV alcohol dependence criteria and exhibited heavy alcohol use patterns before entering treatment: in the 30 days prior to entering treatment, participants drank on an average of $22.8(S D=10.0)$ days, and the mean number of standard drinks a day was $18.9(S D=10.8)$. All participants reported having continuously abstained from psychoactive substance use during their residence in treatment. Reports of abstinence were corroborated by random urinalyses conducted by treatment staff on an as needed basis, as well as through daily observation by program staff.

Participants were recruited after they had resided at least 18 months at the residential facility through an informational presentation about the study, as well as through flyers and direct referrals from facility staff. Study procedures were approved by the University of North Carolina Institutional Review Board. Participants received \$25 for study participation.

\section{Procedure}

On the day of the study, participants first answered demographic questions and then completed two self-report questionnaires, the White Bear Suppression Inventory (Wegner \& Zanakos, 1994) and the Impaired Alcohol Response Inhibition Scale (Guardia, Trujols, Burguete, Luquero, \& Cardus, 2007). Next, they engaged in a computer-based pictorial Addiction-Stroop task. Lastly, participants completed a psychophysiological cue-reactivity protocol. Measures were administered in this same order across participants in a single session.

\section{Measures}

Trait Suppression-Chronic thought suppression, the counterproductive tendency to avoid or suppress undesirable cognitions and emotions, was assessed with the 15-item White Bear Suppression Inventory (WBSI; $\alpha=.84$ ) (Wegner \& Zanakos, 1994). Participants indicated their level of agreement $(1=$ strongly disagree, $5=$ strongly agree $)$ with items such as "I always try to put problems out of mind" and "I often do things to distract myself from 
my thoughts." Items were summed to produce a total score, with higher scores indicative of greater trait thought suppression (in this sample, $M=53.05, S D=9.38$, range $=33$ to 74 ).

Impaired Regulation of Alcohol Urges-Impaired regulation of alcohol urges was measured with the Impaired Response Inhibition Scale (IRISA; $\alpha=.79$ ), a 14-item scale with demonstrated convergent, discriminant, and predictive validity (Guardia et al., 2007). Participants indicated their level of agreement $(1=$ No, never, $4=$ Yes, always $)$ with statements including "When I have decided not to drink, I find it easy not to" and "If I thought about the possibility of drinking I think I could have resisted." Items were summed to produce a total score, with higher scores indicative of more impaired regulation of alcohol urges (in this sample, $M=7.64, S D=5.17$, range $=0$ to 19 ).

Alcohol Attentional Bias-Attentional bias towards alcohol cues was assessed with a pictorial version of the Addiction-Stroop task (Bruce \& Jones, 2004) created in E-Prime 2.0 (PST Inc., Pittsburgh, PA) and presented on an IBM T60 laptop with a 15" screen. On each of 120 trials, one alcohol-related or neutral image appeared in one of three colors: red, green, or blue. Participants were instructed to fixate on the center of the image and indicate the color of the photograph by responding with a press of the correspondingly colored button on a keypad. Reaction times (RTs) were recorded. After a 100ms inter-stimulus interval (ISI), the next photograph was displayed. Photograph content (i.e., alcohol-related or neutral) and color categories were presented randomly with equal frequency. Alcohol stimuli included 13 photographs of alcoholic drinks (liquor, beer, etc.), as well as 7 photos of persons drinking alcohol. Neutral stimuli included 13 photos of kitchen items and 7 photos of persons in kitchen scenes. Stimulus sets were analyzed with respect to their spatial frequency content to ensure they did not differ in basic visual properties. On measures of spectral peak (Neutral: 0.0180, Alcohol: $\left.0.0176, t_{(38)}=0.383, p=0.704\right)$ and width (Neutral: 59.20, Alcohol: $\left.59.29, t_{(38)}=-0.027, p=0.979\right)$, the stimulus sets were not significantly different.

HRV Responsivity to Alcohol Cues-An affect-modulated, cue-reactivity protocol was used to measure HRV responsivity to stress-primed alcohol cues. First, electrocardiogram (ECG) electrodes were attached to participants' right and left pectoral muscles. ECG was sampled at $500 \mathrm{~Hz}$ and recorded continuously throughout the protocol on a Biopac MP150 data acquisition system (Biopac Systems, Goleta, CA). Participants were instructed to remain motionless, silent, and "not think about anything in particular" for a 5-minute baseline. Next, 30 aversive photographs from the International Affective Picture System (IAPS) were serially presented on a 15" laptop screen for 10 seconds each (total duration: 5 $\mathrm{min}$ ) as stress cues. Participants were asked to fixate on the image stream while holding as still as possible. Next, 30 photographs of beer, wine, and distilled liquor (12 of which included individuals drinking or preparing to drink alcohol) were serially presented for 10 seconds each (total duration: $5 \mathrm{~min}$ ), and participants were again instructed to remain still and fixate on the image stream.

Subjective Cue-Reactivity-. During the cue-reactivity protocol, after a 5-minute resting baseline and again after 5 minutes of alcohol cue exposure participants were asked to rate current levels of stress and craving on two, 10-point visual analogue scales (VAS) $(0=$ not at all, $9=$ extreme). Participants were asked the following: "How stressed do you feel right now?" and "If your favorite alcoholic drink were in front of you, how strong of an urge to drink it would you feel right now?"

State Suppression of Thoughts of Drinking-After exposure to the stress-inducing and alcohol-related photographs, participants were asked to rate the degree to which they 
attempted to suppress thoughts about drinking alcohol on a 10-point VAS while viewing the photos with the following question: "How hard did you try to not think about drinking alcohol when looking at the photographs?" Although spontaneous suppression during the cue-reactivity protocol was assessed with this self-report measure, participants were not given specific instructions to suppress thoughts while participating in the protocol.

\section{Data Reduction and Statistical Analyses}

$\mathrm{R}-\mathrm{R}$ intervals (i.e., the time between two consecutive heart beats, represented as R-waves in the ECG) were detected in the ECG data using automated routines in Nevrokard aHRV software (Medistar, Stegne, Ljubljana, Slovenia). The R-wave file was then visually inspected to correct misidentified or omitted R-waves. Kubios 2.0 (Biosignal Analysis and Medical Imaging Group, University of Finland) was used for time-domain analysis of R-R intervals. The square root of the mean squared differences between successive R-R intervals (RMSSD) was selected to estimate vagally-mediated HRV. HRV indices were averaged across the 5-minute baseline and 5-minute alcohol cue-exposure period. The present analysis focused on a particular contrast: HRV responsivity, that is, changes in HRV between baseline and alcohol cue exposure. HRV responsivity was evaluated using an analysis of covariance method, with baseline levels of HRV as a covariate (Manuck, Kasprowicz, Monroe, Larkin, \& Kaplan, 1989).

With regard to the Addiction-Stroop task, RTs of incorrect responses were discarded and the remaining RTs were trimmed (Ratcliffe, 1993); the mean accuracy on the task was .96 (SD $=.04$ ). Trials with extreme RTs ( $>3 \mathrm{SD}$ above the individual mean) were also discarded as outliers (mean number of outliers $=2.2 \pm 1.2$ per participant). The Addiction-Stroop effect was computed as a difference score between RT to neutral photos and RT to alcohol photos, such that higher scores indicate greater attentional bias towards alcohol cues (Bruce \& Jones, 2004).

Paired sample t-tests assessed change in self-reported stress and craving from baseline through alcohol cue-exposure as a measure of subjective cue-reactivity. Subsequently, bivariate and multivariate analyses were conducted to test study hypotheses and explore individual differences in response to the cue-reactivity protocol. Pearson product-moment correlations were used to examine zero-order intercorrelations among trait and state suppression, impaired regulation of alcohol urges, and subjective cue-reactivity. Multiple linear regression was utilized to examine the independent contributions of trait thought suppression, impaired regulation of alcohol urges, and Addiction-Stroop performance to HRV responsivity after controlling for age and number of alcoholic drinks/day before treatment. Model parameters can be interpreted as the extent of prediction of residualized change in HRV, independent of the effects of the covariates. Because prior research has established significant relationships between age and HRV (Koskinen et al., 2009) and quantity of alcohol consumption and HRV (Thayer, Hall, Sollers, \& Fischer, 2006), we controlled for these variables in our HRV analyses. In addition, we ran an additional multiple linear regression model to test for an interaction effect between trait and state suppression on HRV responsivity to alcohol cue-exposure. For all regression analyses, multicollinearity was assessed and ruled-out via examination of tolerance and variance inflation factor statistics.

\section{RESULTS}

\section{Changes in Stress and Craving From Baseline Through Alcohol Cue-Exposure}

Self-reported stress and alcohol craving scores at baseline and alcohol cue-exposure were submitted to paired sample t-tests to assess whether participation in the affect-modulated 
cue-reactivity protocol was associated with increased subjective cue-reactivity. Study participants reported a significant increase in stress and craving from baseline through alcohol cue-exposure $(t(54)=4.81, p<.001 ; t(54)=4.50, p<.001$, respectively).

\section{Pictorial Addiction-Stroop Results}

Paired sample t-tests revealed that subjects were significantly slower to identify the color of alcohol-related photographs (mean RT $=934.9 \mathrm{~ms}, \mathrm{SD}=335.5$ ) than neutral photographs (mean RT $901.0 \mathrm{~ms}, \mathrm{SD}=291.8$ ), $t(54)=3.5, p<.01$, indicating that alcohol cues were more distracting than neutral cues for this sample of adults in residential treatment for alcohol dependence.

\section{Zero-order Correlations Between Impaired Regulation of Alcohol Urges, Trait and State Thought Suppression, and Subjective Cue-Reactivity}

Impaired regulation of alcohol urges was significantly associated with increases in stress $(r$ $=.31, p=.02)$ and craving $(r=.40, p=.003)$ from baseline through alcohol cue exposure. In addition, impaired regulation of alcohol urges was significantly associated with state suppression of thoughts of drinking during alcohol-cue exposure $(r=.51, p<.001)$.

Trait thought suppression was significantly associated with increases in stress $(r=.34, p=$. $01)$ and craving $(r=.38, p=.005)$ from baseline through alcohol cue exposure.

Furthermore, trait thought suppression was significantly associated with state suppression of thoughts of drinking during stress cue-exposure $(r=.30, p<.05)$. State suppression of thoughts of drinking while viewing aversive photographs was significantly associated with increases in stress from baseline to alcohol cue exposure $(r=.41, p=.003)$, but was not associated with changes in craving from baseline to alcohol cue exposure.

\section{Effects of Addiction-Stroop, Thought Suppression, and Impaired Alcohol Response Inhibition on HRV Responsivity}

Inspection of the raw data revealed significant heterogeneity with regard to HRV responsivity. To explicate these individual differences, we tested a multiple linear regression model where Addiction-Stroop, trait thought suppression, and impaired regulation of alcohol urges were entered as predictors of HRV during cue-exposure, controlling for baseline HRV, age, and number of drinks/day. The omnibus F-test for the overall regression model was significant, $F(6,40)=112.52, p<.001, R^{2}=.95$. Multiple linear regression identified significant effects of Addiction-Stroop and impaired regulation of alcohol urges on HRV (see Table 1, model 1), such that higher levels of these variables predicted lower levels of HRV during alcohol cue-exposure. Similarly, a main effect was observed for trait thought suppression on HRV responsivity, indicating that individuals with a greater tendency towards chronic thought suppression had lower HRV during cue-exposure. However, when state suppression of drinking-related thoughts during stress cue-exposure and a state by trait interaction term were added to a second regression model (see Table 1, model $2 ; F(8,40)=$ $94.42, p<.001, R^{2}=.96$ ), this effect was qualified by a significant state $\mathrm{X}$ trait suppression interaction on HRV responsivity. Figure 2 displays predicted HRV values during cueexposure at $1 \mathrm{SD}$ above and below the centered state and trait suppression means for the interaction. Alcohol dependent individuals with high trait thought suppression who engaged in high levels of state suppression of thoughts of drinking during stress cue-exposure exhibited less HRV responsivity than persons low in both trait and state thought suppression. In contrast, alcohol dependent adults low in trait thought suppression who engaged in high levels of state suppression evidenced comparatively greater HRV responsivity than persons who reported high levels of state and trait thought suppression. 


\section{DISCUSSION}

Study findings indicated that alcohol dependent adults who evidenced higher levels of trait thought suppression, impaired regulation of alcohol urges, and Addiction-Stroop effects exhibited less HRV responsivity to stress-primed alcohol cues. Hence, among persons with a history of alcohol dependence, individual differences in HRV responsivity to alcohol cues were significantly related to degree of alcohol attentional bias, the tendency towards trait thought suppression as a coping response, and a perceived inability to regulate drinking urges. These factors appear co-extensive and may be conceptually linked to deficient inhibitory processes subserved by the central autonomic network. Moreover, trait and state thought suppression, which were associated with self-reported stress and craving during the affect-modulated cue-reactivity protocol, significantly interacted to affect HRV responsivity.

Current findings are congruent with the investigation conducted by Ingjaldsson et al. (2003), who found significant relationships between trait thought suppression, inability to regulate alcohol urges, and HRV responsivity to alcohol cues. The present work replicates earlier findings by identifying relationships between trait thought suppression and impaired regulation of alcohol urges on HRV responsivity to alcohol cues. Extending these findings, the current investigation examined the interactive effects of trait and state thought suppression on HRV cueresponsivity, and used a pictorial Addiction-Stroop task to detect the presence of a significant alcohol AB that was associated with HRV responsivity to alcohol cues. Furthermore, we established these relationships within an affect-modulated cue-reactivity paradigm. This paradigm, wherein the presentation of alcohol cues was primed by exposure to stressful stimuli, may serve as a proxy for real-world situations where stress exposure promotes the occurrence of drinking urges (Sinha, 2007).

The identified relation between trait thought suppression, subjective cue-reactivity, and HRV responsivity to stress-primed alcohol cues is conceptually meaningful given the known ineffectiveness of thought suppression as a method of achieving control over undesired mental states (Abramowitz et al., 2001; Wenzlaff \& Wegner, 2000) and behavior (Erskine et al., 2010; Denzler, Förster, Liberman, \& Rozenman, 2010). Indeed, the attempt to suppress unwanted thoughts and feelings may result in rebound effects that are more difficult to regulate than the original targets of suppression. Possibly indicative of such rebound effects, alcohol dependent patients endorsing greater trait thought suppression experienced larger increases in stress and craving from baseline through alcohol cue-exposure and less HRV responsivity than patients with reduced propensity towards thought suppression.

Moreover, persons evidencing higher levels of trait thought suppression reported trying harder to suppress thoughts of drinking during stress cue-exposure. Such elevated state suppression predicted larger increases in subjective stress from baseline through alcohol cue-exposure. Concomitantly, the interaction of trait thought suppression and state suppression of alcohol-related thoughts during stress cue-exposure had significant effects on HRV. High trait thought suppressors who engaged in more intense state suppression of drinking-related thoughts while viewing stressful photographs evidenced less HRV during alcohol cue-exposure than low trait suppressors who engaged in less intense state suppression of drinking-related thoughts while viewing the same photographs. If suppression exhausts self-regulatory resources, and HRV is an index of self-regulatory capacity, then diminished HRV responsivity associated with the interaction of trait and state suppression may reflect the exhaustion of chronically taxed regulatory resources over the course of coping with the series of aversive and alcohol-related images, a phenomena predicted by theories of self-control as a limited resource (Baumeister, 2003; Muraven \& Shmueli, 2006). In partial support of this interpretation, suppression of negative emotion associated with individual differences in HRV has been shown to lead to transient decreases 
in executive function, as evidenced by reduced working memory performance (Pu et al., 2010). As such, among high trait thought suppressors in the present study, suppression of emotional responses to aversive stimuli may have depleted the cognitive resources needed for emotion regulation (Ochsner \& Gross, 2005), resulting in an inability to manage affective distress arising from viewing stress-primed alcohol cues.

In contrast, low trait thought suppressors who engaged in higher levels of state suppression of alcohol urges during stress-cue exposure tended to exhibit higher levels of HRV responsivity to alcohol cues. The effects of state suppression on HRV may reflect central autonomic network activity during emotion regulatory efforts, that, when inflexibly maintained, becomes maladaptive. Other research has documented time-limited increases in HRV to emotional challenges during state suppression (Butler, Wilhelm, \& Gross, 2006). Given theories of self-control as a limited resource (Baumesiter, 2003), we hypothesize that state suppression during an emotional provocation would elicit an initial, transient increase in HRV followed by a decrease in HRV when suppression is prolonged until self-regulatory resources are depleted.

Independent of trait thought suppression, persons endorsing more severe impairment in urge regulation experienced elevated stress and reduced HRV responsivity from baseline to cueexposure in comparison to their counterparts reporting less severe impairment in urge regulation. These findings may be informed by Porges' (1995) conceptualization of vagallymediated heart rate variability during stress as an index of adaptation to environmental demands. According to this conceptualization, changes in vagal activity modulate visceral state to allow for effective regulation of emotion and adaptive engagement and disengagement with motivationally-salient objects and events (Porges, 1995). Thus, in the present study, alcohol dependent inpatients with a sense of inefficacy over drinking urges appeared to have difficulty adapting to the emotional challenge presented by stress and alcohol cues, as reflected by their attenuated parasympathetic cue-responses and concomitant affective distress.

Taken together, study results suggest that alcohol dependent persons reporting high levels of thought suppression and impaired regulation of alcohol urges exhibit a deficit in autonomic flexibility and inhibitory capacity (Friedman, 2007). Indeed, low levels of HRV are associated with poor executive function, deficient attentional control, and limited working memory (Thayer et al., 2009), as well an inability to successfully regulate negative emotions (Bleil, Gianaros, Jennings, Flory, \& Manuck, 2008; Thayer \& Lane, 2000). Moreover, perseverative cognition, such as that which may be engendered by the rebound effects of suppression, has been characterized as a failure of inhibition subserved by dysfunction of the central autonomic network (Thayer \& Friedman, 2002). The inhibitory deficits observed among alcohol dependent individuals reporting greater impaired regulation of alcohol urges and trait thought suppression may increase risk of relapse under conditions of stress. When adverse life circumstances trigger perseveration on the stressor and alcohol as a means of coping, such perseveration cognition unchecked by efficacious inhibitory mechanisms may lead to prolonged stress activation (Brosschot, Pieper, \& Thayer, 2005). In turn, ineffective coping with the sequelae of stress via suppression depletes the resources needed for selfregulation (Muraven \& Baumeister, 2000), rendering the alcohol dependent individual vulnerable to drinking urges and leading to increased consumption of alcohol (Baumeister, 2003; Muraven, Collins, \& Nienhaus, 2002).

In the present study, individuals whose attention was highly biased toward alcohol cues on the Addiction-Stroop task exhibited attenuated HRV responsivity to alcohol cue-exposure. Thus, deficient neurocognitive inhibitory capacity as indexed by lower HRV responsivity to alcohol cues was significantly associated with alcohol attentional bias. This identified 
relation between inhibition and attention is consistent with cognitive neuroscience models of attention as a form of biased competition (e.g., Desimone \& Duncan, 1995), wherein attention is thought to allow certain subsets of information to gain preeminence in the competitive processing of neural networks via the inhibition of other less salient information. Given the heightened incentive salience of alcohol cues conferred by dopamingeric neuroadaptations occurring during the transition from alcohol use to dependence (Robinson \& Berridge, 2008), alcohol-related stimuli would tend to automatically captivate attention especially among persons who are less able to inhibit information unrelated to their current concerns (Cox, Fadardi, \& Pothos, 2006). Further accentuating the attentional bias driven by "bottom-up" neurobiological processes of incentive sensitization, the attempt to suppress a thought invokes an automatized search for the target of suppression that raises the thought's activation level and increases its accessibility to consciousness (Wegner \& Erber, 1992). Insofar as highly accessible concepts are more likely to be enacted in behavior, even without conscious intent (e.g., Bargh, Chen, \& Burrows, 1996), suppression coupled with attentional bias may undermine the inhibition of alcohol urges and foster appetitive behavioral responses.

Based on our theoretical model depicted in Figure 1, we offer the following description of how the processes explored in the current study might play out in a "real world" scenario. A recovering alcohol dependent person who chronically engages in thought suppression may pass by a bar on his way home from a stressful day at the office, and attempt to suppress the urge to drink. Inadvertently, this state suppression evokes an automatic search for thoughts of drinking, taxing his inhibitory system farther and making it difficult to maintain attention on the goal (returning home to his family without drinking) after priming by stress and alcohol cues. Over the course of minutes, self-control resources are depleted by suppression while thoughts of drinking become hyperaccessible, resulting in a rebound of alcohol-related cognitions and urges which may overwhelm inhibitory mechanisms. Consequently, attention becomes fixated on alcohol cues and internal representations of such cues until the individual makes a detour into the parking lot of a nearby liquor store - the site of his next relapse.

The present study is limited by a number of factors. First, its cross-sectional design employed precludes causal inferences. Without experimental induction of thought suppression, we cannot ascertain whether the trait and state thought suppression measured in the present study is the cause, correlate, or consequence of the attenuated HRV response observed. However, this limitation is partially offset by our data on the prospective association between state suppression during stress cue-exposure and stress and HRV during alcohol cue-exposure. Similarly, the current study design cannot fully test the theoretical model depicted in Figure 1, nor can it probe causal relationships between trait and state thought suppression, impaired regulation of alcohol urges, the Addiction-Stroop effect, and HRV responsivity. In addition, given the long length of abstinence from alcohol among study participants, current study findings may not be generalizable to current drinkers or persons early in recovery from alcohol dependence. Also, interpretations of HRV data may be confounded because we were unable to control for the effects of respiration (Grossman \& Taylor, 2007), although there is significant debate in the literature regarding the need for such corrections (e.g., Denver, Reed, \& Porges, 2007). Finally, because the presentation order of stress and alcohol cues was not counterbalanced, the differential contribution of each type of cue to affective and physiological responses cannot be ascertained. As such, current study findings are subject to alternative explanations that should be investigated and potentially ruled-out in future studies. To redress these limitations, work is ongoing to replicate these findings in a much larger, randomly-selected sample of recently abstinent alcohol dependent persons who will be followed for several years. 
In conclusion, study findings suggest that among recovering alcohol dependent persons, trait suppression of unwanted thoughts and feelings, the perceived inability to inhibit alcohol urges, and the biasing of attention toward alcohol cues are closely linked with impaired regulation of cue-reactivity as indexed by diminished HRV responsivity. Further, when recovering individuals who report strong tendencies toward trait thought suppression attempt to suppress thoughts of drinking triggered by stressors, they exhibit physiological responses to alcohol cues that render them especially vulnerable to relapse. Such persons may benefit from mindfulness-based addictions treatment, which provides instruction in coping with stress and alcohol urges through metacognition and acceptance rather than suppression, while targeting the pathogenic attentional, affective, and autonomic mechanisms that contribute to relapse (Garland et al., 2011). Indeed, a recent randomized controlled trial of a mindfulness-oriented recovery enhancement intervention conducted by Garland and colleagues (2010) identified significant therapeutic effects on thought suppression that were correlated with decreased alcohol attentional bias and increased HRV recovery from alcohol cues. Treatment approaches offering effective alternatives to the maladaptive strategy of suppressing the urge to drink in the face of relapse triggers may enable the central autonomic network to disengage from perseveration on alcohol, thereby freeing neurocognitive resources for the regulation of emotional distress and concomitant urges (Garland, in press). Facilitation of regulatory mechanisms in alcohol dependent individuals appears to be an important key to recovering from the perturbations to homeostasis induced by exposure to alcohol cues in the natural environment.

\section{Acknowledgments}

Data collection for this project was supported by Grant Number T32AT003378 from the National Center for Complementary and Alternative Medicine, a Francisco J. Varela Research Grant from the Mind and Life Institute, Boulder, CO, and an Armfield-Reeves Innovation Grant from the University of North Carolina, Chapel Hill, NC awarded to the first author.

\section{REFERENCES}

Abramowitz JS, Tolin DF, Street GP. Paradoxical effects of thought suppression: A meta-analysis of controlled studies. Clinical Psychology Review. 2001; 21:683-703. [PubMed: 11434226]

American Psychiatric Association. Diagnostic and statistical manual of mental disorders. 4th ed., Text Revision. Washington, DC: 2000.

Bargh JA, Chen M, Burrows L. Automaticity of social behavior: Direct effects of trait construct and stereotype activation on action. Journal of Personality and Social Psychology. 1996; 71:230-244. [PubMed: 8765481]

Baumeister RF. Ego depletion and self-regulation failure: A resource model of self-control. Alcoholism: Clinical \& Experimental Research. 2003; 27:281-284.

Bleil ME, Gianaros PJ, Jennings JR, Flory JD, Manuck SB. Trait negative affect: Toward an integrated model of understanding psychological risk for impairment in cardiac autonomic function. Psychosomatic Medicine. 2008; 70:328-337. [PubMed: 18378862]

Brosschot JF, Gerin W, Thayer JF. The perseverative cognition hypothesis: A review of worry, prolonged stress-related physiological activation, and health. Journal of Psychosomatic Research. 2006; 60:113-124. [PubMed: 16439263]

Brosschot JF, Pieper S, Thayer JF. Expanding stress theory: Prolonged activation and perseverative cognition. Psychoneuroendocrinology. 2005; 30:1043-1049. [PubMed: 15939546]

Brosschot JF. Markers of chronic stress: Prolonged physiological activation and (un)conscious perseverative cognition. Neuroscience and Biobehavioral Reviews. 2010; 35:46-50. [PubMed: 20096302]

Bruce G, Jones BT. A pictorial Stroop paradigm reveals an alcohol attentional bias in heavier compared to lighter social drinkers. Journal of Psychopharmacology. 2004; 18:527-533. [PubMed: 15582919] 
Butler EA, Wilhelm FH, Gross JJ. Respiratory sinus arrhythmia, emotion, and emotion regulation during social interaction. Psychophysiology. 2006; 43:612-622. [PubMed: 17076818]

Cappell H, Herman CP. Alcohol and tension reduction. A review. Quarterly Journal of Studies on Alcohol. 1972; 33:33-64. [PubMed: 4551021]

Conger JJ. The effects of alcohol on conflict behavior in the albino rat. Quarterly Journal of Studies on Alcohol. 1951; 12:1-29. [PubMed: 14828044]

Cheetham A, Allen NB, Yucel M, Lubman DI. The role of affective dysregulation in drug addiction. Clinical Psychology Review. 2010; 30:621-634. [PubMed: 20546986]

Cox WM, Fadardi JS, Pothos EM. The Addiction-Stroop test: Theoretical considerations and procedural recommendations. Psychological Bulletin. 2006; 132:443-476. [PubMed: 16719569]

Denver JW, Reed SF, Porges SW. Methodological issues in the quantification of respiratory sinus arrhythmia. Biological Psychology. 2007; 74:286-294. [PubMed: 17067734]

Denzler M, Förster J, Liberman N, Rozenman M. Aggressive, funny, and thirsty: A Motivational Inference Model (MIMO) approach to behavioural rebound. Personality and Social Psychology Bulletin. 2010; 36:1385-1396. [PubMed: 20823297]

Desimone R, Duncan J. Neural mechanisms of selective visual attention. Annual Review of Neuroscience. 1995; 18:193-222.

Dias-Ferreira E, Sousa JC, Melo I, Morgado P, Mesquita AR, Cerqueira JJ, Costa RM, Sousa N. Chronic stress causes frontostriatal reorganization and affects decision-making. Science. 2009; 325:621-625. [PubMed: 19644122]

Erskine JA, Georgiou GJ. Effects of thought suppression on eating behaviour in restrained and nonrestrained eaters. Appetite. 2010; 54:499-503. [PubMed: 20152872]

Erskine JA, Georgiou GJ, Kvavilashvili L. I think therefore I smoke. Psychological Science. 2010; 21:1225-1230. [PubMed: 20660892]

Field M, Powell H. Stress increases attentional bias for alcohol cues in social drinkers who drink to cope. Alcohol and Alcoholism. 2007; 42:560-566. [PubMed: 17766316]

Friedman BH. An autonomic flexibility-neurovisceral integration model of anxiety and cardiac vagal tone. Biological Psychology. 2007; 74:185-199. [PubMed: 17069959]

Garland EL, Gaylord SA, Boettiger CA, Howard MO. Mindfulness training modifies cognitive, affective, and physiological mechanisms implicated in alcohol dependence: Results from a randomized controlled pilot trial. Journal of Psychoactive Drugs. 2010; 42:177-192. [PubMed: 20648913]

Garland EL, Boettiger CA, Howard MO. Targeting cognitive-affective risk mechanisms in stressprecipitated alcohol dependence: An integrated, biopsychosocial model of allostasis, automaticity, and addiction. Medical Hypotheses. 2011; 76:745-754. [PubMed: 21354711]

Garland EL. Trait mindfulness predicts attentional and autonomic regulation of alcohol cue-reactivity. Journal of Psychophysiology. in press.

Gross J, Levenson R. Emotional suppression: Physiological, self-report, and expressive behavior. Journal of Personality and Social Psychology. 1993; 64:970-986. [PubMed: 8326473]

Grossman P, Taylor EW. Toward understanding respiratory sinus arrhythmia: Relations to cardiac vagal tone, evolution and biobehavioral functions. Biological Psychology. 2007; 74:263-285. [PubMed: 17081672]

Guardia J, Trujols J, Burquete T, Luquero E, Cardus M. Impaired response inhibition scale for alcoholism (IRISA): Development and psychometric properties of a new scale for abstinenceoriented treatment of alcoholism. Alcoholism: Clinical and Experimental Research. 2007; 31:269275.

Hansen AL, Johnsen BH, Thayer JF. Vagal influence on working memory and attention. International Journal of Psychophysiology. 2003; 48:263-274. [PubMed: 12798986]

Ingjaldsson JT, Laberg JC, Thayer JF. Reduced heart rate variability in chronic alcohol abuse: Relationship with negative mood, chronic thought suppression, and compulsive drinking. Biological Psychiatry. 2003; 54:1427-1436. [PubMed: 14675808]

Kavanagh D, May J, Andrade J. Tests of the elaborated intrusion theory of craving and desire: Features of alcohol craving during treatment for an alcohol disorder. British Journal of Clinical Psychology. 2009; 48:241-254. [PubMed: 19364447] 
Key BL, Campbell TS, Bacon SL, Gerin W. The influence of trait and state rumination on cardiovascular recovery from a negative emotional stressor. Journal of Behavior Medicine. 2008; 31:237-248.

Klein AA. Suppression-induced hyperaccessibility of thoughts in abstinent alcoholics: a preliminary investigation. Behavior Research \& Therapy. 2007; 45:169-177.

Koskinen T, Kahonen M, Jula A, Laitinen T, Keltikangas-Jarvinen L, Viikari J, Valimaki I, Raitakari OT. Short-term heart rate variability in healthy young adults: the Cardiovascular Risk in Young Finns Study. Autonomic Neuroscience. 2009; 145:81-88. [PubMed: 19019739]

Manuck, SB.; Kasprowicz, AL.; Monroe, SM.; Larkin, KT.; Kaplan, JR. Psychophysiologic reactivity as a dimension of individual differences.. In: Schneiderman, N.; Weiss, SM.; Kaufmann, PG., editors. Handbook of Research Methods in Cardiovascular Behavioral Medicine. Plenum; New York: 1989. p. 365-382.

Muraven M, Baumeister RF. Self-regulation and depletion of limited resources: does self-control resemble a muscle? Psychological Bulletin. 2000; 126:247-259. [PubMed: 10748642]

Muraven M, Collins RL, Nienhaus K. Self-control and alcohol restraint: an initial application of the self-control strength model. Psychology of Addictive Behaviors. 2002; 16:113-120. [PubMed: 12079249]

Muraven M, Shmueli D. The self-control costs of fighting the temptation to drink. Psychology of Addictive Behaviors. 2006; 20:154-160. [PubMed: 16784361]

Nixon RD, Cain N, Nehmy T, Seymour M. The influence of thought suppression and cognitive load on intrusions and memory processes following an analogue stressor. Behavior Therapy. 2009; 40:368-379. [PubMed: 19892082]

Ochsner KN, Gross JJ. The cognitive control of emotion. Trends in Cognitive Sciences. 2005; 9:242249. [PubMed: 15866151]

Palfai TP, Monti PM, Colby SM, Rohsenow DJ. Effects of suppressing the urge to drink on the accessibility of alcohol outcome expectancies. Behaviour Research and Therapy. 1997; 35:59-65. [PubMed: 9009044]

Porges SW. Orienting in a defensive world: Mammalian modifications of our evolutionary heritage. A Polyvagal theory. Psychophysiology. 1995; 32:301-318. [PubMed: 7652107]

$\mathrm{Pu}$ J, Schmeichel BJ, Demaree HA. Cardiac vagal control predicts spontaneous regulation of negative emotional expression and subsequent cognitive performance. Biological Psychology. 2010; 84:531-540.

Ratcliffe R. Methods of dealing with reaction-time outliers. Psychological Bulletin. 1993; 114:510 532. [PubMed: 8272468]

Roberts N, Levenson R, Gross J. Cardiovascular costs of emotion suppression cross ethnic lines. International Journal if Psychophysiology. 2008; 70:82-87.

Robinson TE, Berridge KC. Review. The incentive sensitization theory of addiction: some current issues. Philosophical Transactions of the Royal Society of London, B Biological Sciences. 2008; 363:3137-3146.

Sinha R. The role of stress in addiction relapse. Current Psychiatry Reports. 2007; 9:388-395. [PubMed: 17915078]

Soutullo CA, McElroy SL, Goldsmith JR. Cravings and irresistible impulses: Similarities between addictions and impulse control disorders. Psychiatric Annals. 1998; 28:592-600.

Thayer JF, Hansen AL, Saus-Rose E, Johnsen BH. Heart rate variability, prefrontal neural function, and cognitive performance: The neurovisceral integration perspective on self-regulation, adaptation, and health. Annals of Behavioral Medicine. 2009; 37:141-153. [PubMed: 19424767]

Thayer JF, Hall M, Sollers JJ 3rd, Fischer JE. Alcohol use, urinary cortisol, and heart rate variability in apparently healthy men: Evidence for impaired inhibitory control of the HPA axis in heavy drinkers. International Journal of Psychophysiology. 2006; 59:244-250. [PubMed: 16325293]

Thayer JF, Lane RD. A model of neurovisceral integration in emotion regulation and dysregulation. Journal of Affective Disorders. 2000; 61:201-216. [PubMed: 11163422]

Thayer JF, Friedman BH. Stop that! Inhibition, sensitization, and their neurovisceral concomitants. Scandinavian Journal of Psychology. 2002; 43:123-130. [PubMed: 12004949] 
Tiffany ST. A cognitive model of drug urges and drug-use behavior: Role of automatic and nonautomatic processes. Psychological Review. 1990; 97:147-168. [PubMed: 2186423]

Wegner DM, Erber R. The hyperaccessibility of suppressed thoughts. Journal of Personality and Social Psychology. 1992; 63:903-912.

Wegner DM. Ironic processes of mental control. Psychological Review. 1994; 101:34-52. [PubMed: 8121959]

Wegner D, Schneider D, Carter S, White T. Paradoxical effects of thought suppression. Journal of Personality and Social Psychology. 1987; 53:5-13. [PubMed: 3612492]

Wegner DM, Zanakos S. Chronic thought suppression. Journal of Personality. 1994; 62:616-640. [PubMed: 7861307]

Wenzlaff RM, Wegner DM. Thought suppression. Annual Review of Psychology. 2000; 51:59-91.

Williams AD, Moulds ML. Investigation of the indulgence cycles hypothesis of suppression on experimentally induced visual intrusions in dysphoria. Behavior Research \& Therapy. 2007; 45:2780-2788. 


\section{Research Highlights}

- Thought suppression is associated with deficient HRV response to alcohol cues.

- Impaired regulation of urges is associated with deficient HRV response to alcohol cues.

- Addiction Stroop is associated with deficient HRV response to alcohol cues.

- State suppression exhausts cognitive control resources reflected in stress and HRV. 


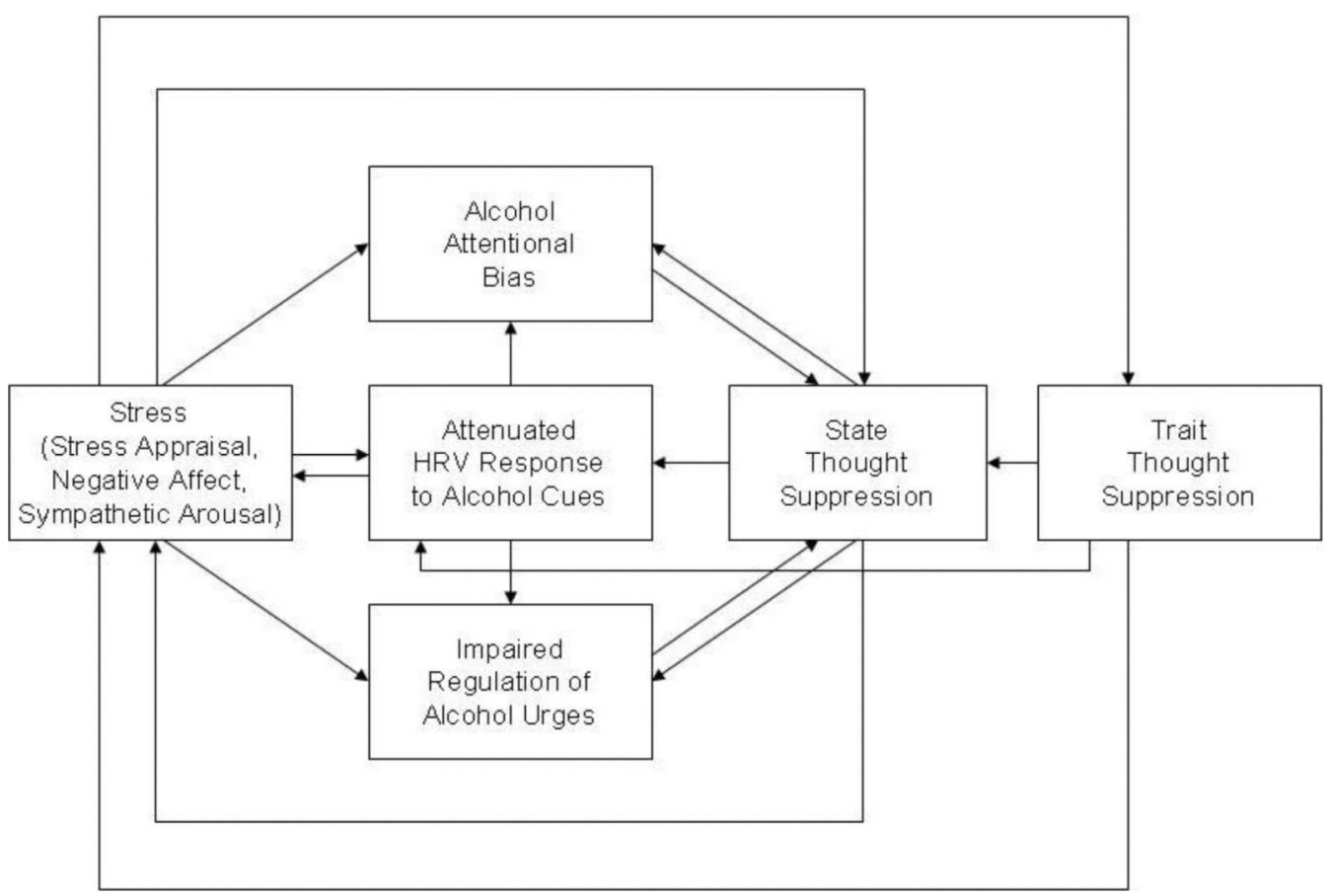

Figure 1.

Theoretical Model Depicting Relationships Between Stress, Trait and State Thought Suppression, Impaired Regulation of Alcohol Urges, Alcohol Attentional Bias, and Attenuated HRV Response to Alcohol Cues. 

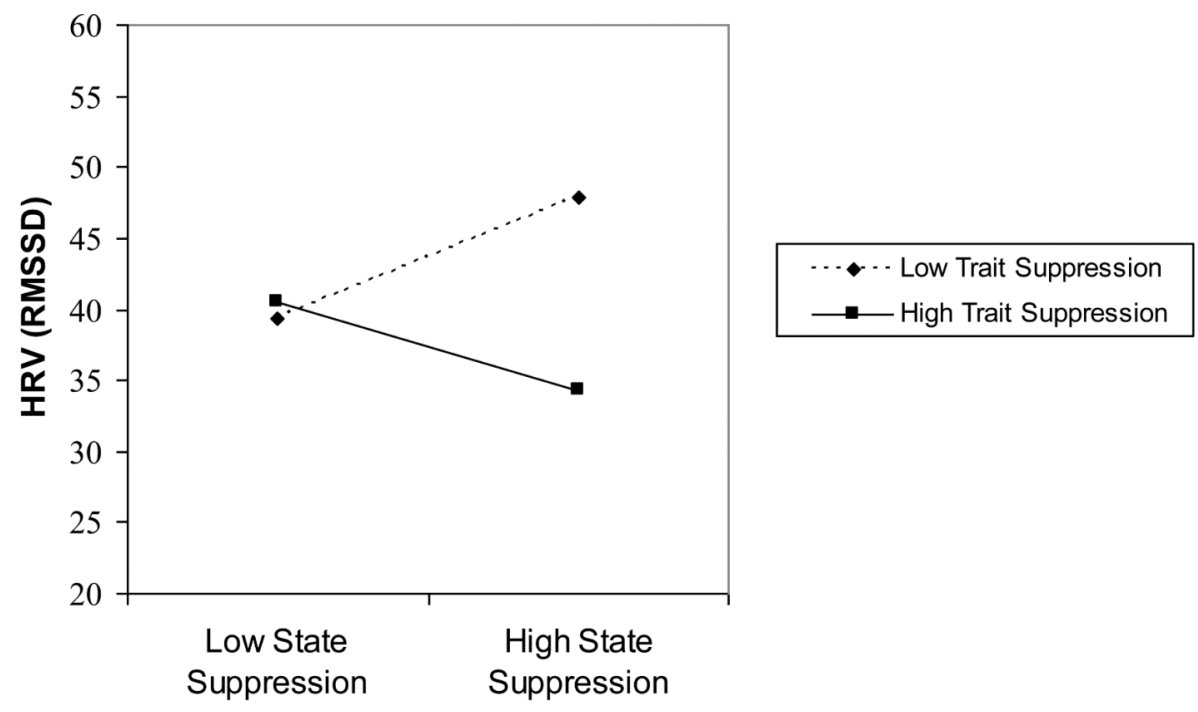

a Values plotted from a multiple regression equation controlling for the main and interaction effects of state
and trait thought suppression, as well as baseline HRV, Addiction-Stroop, impaired urge regulation, age,
and number of drinks/day. RMSSD = root-mean square of successive differences in R-R intervals.

Figure 2.

Interaction between state and trait thought suppression on HRV values during alcohol cueexposure $^{\mathrm{a}}$. Simple slopes reflect predicted values at 1 standard deviation above and below the centered mean for state and trait suppression. 


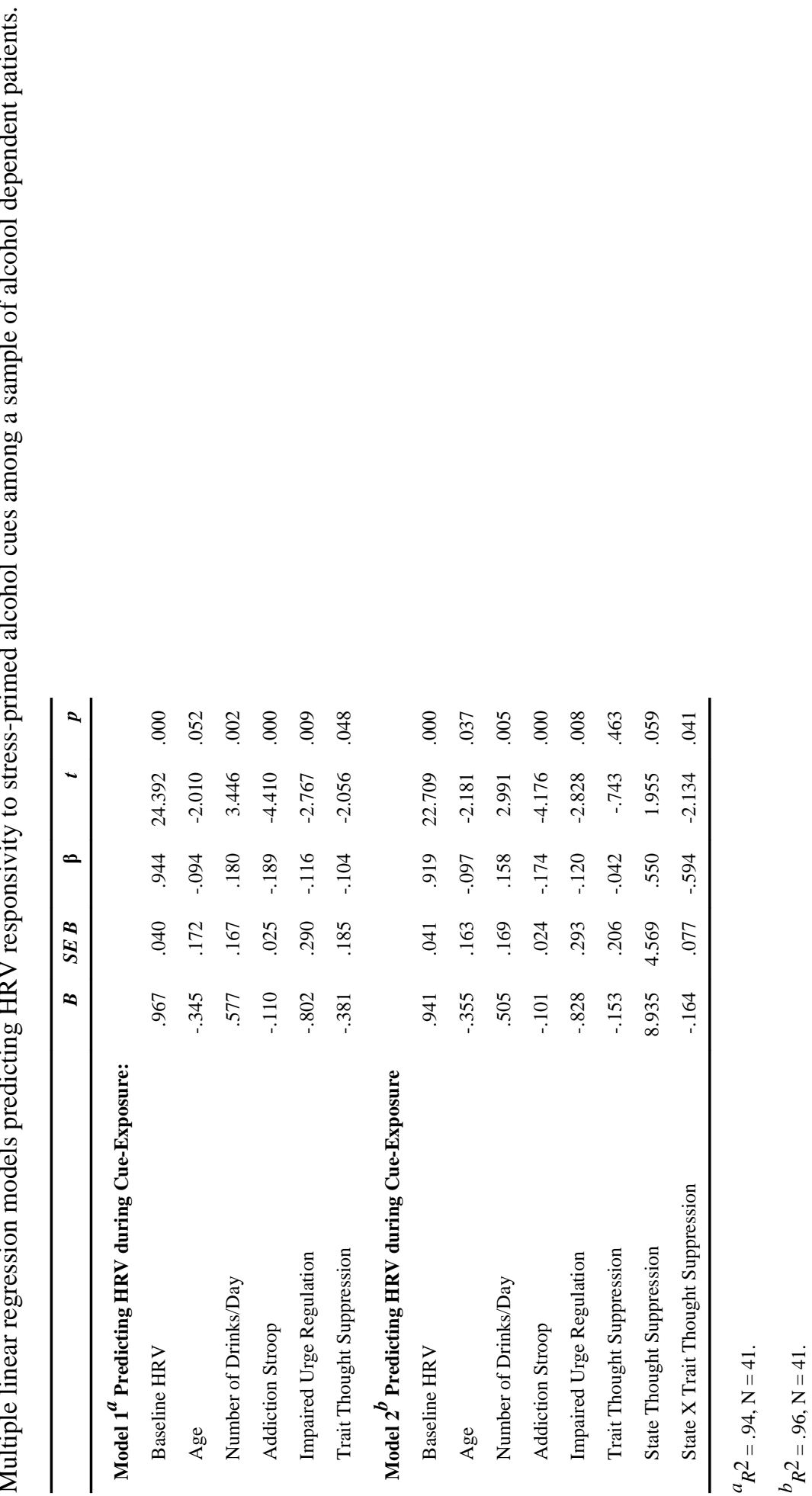

